\title{
Pedigree analysis, diagnosis and treatment in Von Hippel-Lindau syndrome: A report of three cases
}

\author{
YUANLIANG WANG ${ }^{1}$, GUOBIAO LIANG $^{1 *}$, JING TIAN $^{2 *}$, XIN WANG $^{1 *}$, ANJIAN CHEN $^{1}$, \\ TIANCAI LIANG ${ }^{1}$, YANG DU ${ }^{1}$, HAO LI $^{1}$, JIANG DU ${ }^{1}$, LANG YU ${ }^{1}$ and ZONGPING CHEN ${ }^{1}$ \\ Departments of ${ }^{1}$ Urology and ${ }^{2}$ Anesthesiology, Affiliated Hospital of Zunyi Medical College, \\ Zunyi, Guizhou 563000, P.R. China
}

Received February 19, 2016; Accepted February 17, 2017

DOI: 10.3892/ol.2018.7957

\begin{abstract}
The objective of the present study was to systematically investigate the clinical features, diagnosis and therapeutic treatment of Von Hippel-Lindau (VHL) syndrome in order to improve understanding of this disease. A total of 3 cases of VHL syndrome treated at the Affiliated Hospital of Zunyi Medical College (Zunyi, China) between September 2014 and October 2015 were retrospectively analyzed. The associated literature was reviewed, and the diagnostic and therapeutic features were discussed. Case 1 was diagnosed as VHL syndrome accompanied by a renal tumor on the right side, and radical tumor resection in the right kidney was performed. Postoperative pathological examination indicated clear cell carcinoma. Case 2 was diagnosed as VHL syndrome accompanied by bilateral adrenal pheochromocytoma. The left-side adrenal tumor was removed, and postoperative pathological analysis was suggestive of adrenal pheochromocytoma. Case 3 visited the hospital due to the presence of masses on the left and right sides of the kidney, but did not undergo surgery for personal reasons. Follow-ups were scheduled subsequent to surgery at another hospital. The diagnosis in all 3 cases was confirmed by genetic testing, where VHL mutations were detected in all patients. Following surgery, pedigree and genetic analysis was performed in all 3 pedigrees and VHL mutations were identified in 7 family members. The diagnosis of VHL syndrome should be based on the clinical manifestation of the patients and the results of genetic tests. DNA analysis of mutations is the main method for diagnosis. An appropriate surgical plan should be formulated based on the site, size and number of tumors, and the condition of the
\end{abstract}

Correspondence to: Dr Guobiao Liang, Department of Urology, Affiliated Hospital of Zunyi Medical College, 149 Dalian Road, Zunyi, Guizhou 563000, P.R. China

E-mail: lgb1111@126.com

*Contributed equally

Key words: von Hippel-Lindau syndrome, diagnosis, therapeutics, pedigree analysis, genetic test patient. Since VHL syndrome is an inheritable genetic disorder and relapse following surgery is common, pedigree analysis of the patient and lifelong follow-ups are essential. Additionally, physicians should pay attention to VHL syndrome in order to avoid missing diagnosis or misdiagnosis.

\section{Introduction}

Von Hippel-Lindau (VHL) syndrome is an autosomal dominant genetic disorder with an incidence of $1 / 36,000$ to $1 / 45,500$ (1). VHL syndrome is characterized by hemangioblastoma of the central nervous system (CNS) and retina, and multiple cysts and tumors in visceral organs. VHL syndrome may occur at any age, and the penetrance is $>90 \%$ prior to the age of 65 (1). Without treatment, most patients succumb to the disease by the age of 50 years, and the most common causes of VHL syndrome-associated mortality are comorbidities of cerebellar hemangioblastoma or metastasis of renal cell carcinoma (2). In 1993, Latif et al (3) reported that the VHL gene was located on chromosome $3 \mathrm{p} 25-26$ by linkage analysis, and for the first time the VHL gene was successfully cloned. The VHL gene is a tumor suppressor. The encoded protein is able to regulate the activity of elongin, a transcription elongation factor, and suppress tumor growth by inhibiting mRNA synthesis (4).

Mutation, deletion or methylation of the VHL gene may lead to abnormal protein synthesis. Therefore, VHL syndrome is associated with disruption of VHL gene structure or function (4). According to whether the patient develops pheochromocytoma or renal carcinoma, VHL syndrome may be divided into types I and II. Pheochromocytoma is only present in type II VHL syndrome (5). These subtypes may be further divided. Type IA is characterized by retinal and CNS hemangioblastoma, pancreatic cysts, neuroendocrine tumors and renal carcinoma. Type IB is characterized by retinal and CNS hemangioblastoma, pancreatic cysts and neuroendocrine tumors without the presence of renal carcinoma. Type IIA is characterized by pheochromocytoma and retinal and CNS hemangioblastoma without the presence of renal carcinoma. Pheochromocytoma, retinal and CNS hemangioblastoma, as well as renal carcinoma, are present in type IIB VHL syndrome. Patients presenting only with pheochromocytoma are classified as type $2 \mathrm{C}(5)$. Based on the classification criteria, the 3 cases of VHL syndrome treated at the Affiliated Hospital 
of Zunyi Medical College (Zunyi, China) between September 2014 and October 2015 were characterized as type IA, $2 \mathrm{~A}$ and IA, repectively. The present study was approved by the Medical Ethics Committee at the Affiliated Hospital of Zunyi Medical College and written informed consent was obtained from all patients.

\section{Case report}

Case 1. On September 10, 2014, a 38-year-old male patient visited the Department of Urology at the Affiliated Hospital of Zunyi Medical College due to the detection of lesions in his right kidney during a physical examination. In November 2011, the patient visited Chongqing Southwest Hospital (Chongqing, China) due to dizziness and headache. Lesions were detected in the left side of the brain stem (Fig. 1A). The tumor was removed by surgery. Postoperative pathological examination confirmed that the lesions were hemangioblastomas. VHL syndrome was not considered as a diagnosis at that time. The patient had no previous history of hypertension, infectious diseases or allergy. The patient's mother was deceased, and the cause may have been associated with VHL. The patient's father suffered from hypertension and diabetes. One of the patient's sisters (II-3) suffered from diabetes, and the remaining siblings had no history of hypertension, diabetes or tumor.

Physical examination after admission demonstrated that the body temperature of the patient was $36.5^{\circ} \mathrm{C}$, and the heart rate was 81 beats $/ \mathrm{min}$. The respiration rate of the patient was 18 times/min, and blood pressure was $132 / 94 \mathrm{mmHg}$. The patient exhibited normal development, slow gait and poor balance. Old surgical scars were observed on the left side of the occipital region. Muscle atrophy was observed to be marginally more on the left side of the limbs compared with the contralateral limbs. The patient responded well in terms of sensation, pain and physiological reflex. No abnormalities were observed in the heart, lung or abdomen. Bilateral renal computed tomography (CT) indicated the presence of a $64 \times 59 \mathrm{~mm}$ mass in the right renal parenchyma, with a clear edge. The mass protruded into the renal capsule and suppressed the renal pelvis. Low-density areas with multiple cysts and shadows of line-like partitions were observed inside the mass. Contrast-enhanced scanning revealed uneven enhancement, and the partitions demonstrated significant enhancement. In delayed-phase imaging, the contrast was strengthened and patch-like intensified shadows were observed in the middle of the mass (Fig. 1B).

Brain CT revealed that the left occipital bone was altered following surgery. Malacia was observed in the left side of the cerebellum. Multiple enhanced nodules were observed in the brain. Brain magnetic resonance imaging (MRI) indicated that bone defect and the shadow of the internal fixation were observed in the left occipital bone. Weak long T2 signals were observed in the left cerebellar hemisphere. Enhanced nodules were observed in the right occipital lobe, right cerebellar hemisphere and foramen magnum. Weak long T2 signals could be observed in the surrounding areas. The largest nodule was located in the right cerebellar hemisphere with a size of $\sim 8 \times 10 \mathrm{~mm}$ (Fig. 1C). MRI of the thoracolumbar spinal cord revealed that multiple nodule-like shadows were present on the surface of the thoracolumbar spinal cord and nerve plexus in the cauda equina. The larger nodules had a diameter of $\sim 6 \mathrm{~mm}$. The contrast was markedly enhanced in the MRI images. Abnormal enlarged blood vessels were observed in the surrounding areas, which appeared to indicate hemangioma. Multiple nodules with signals of a similar intensity were observed next to the lumbar spinal canal and cauda equina. The larger nodules had a diameter of $\sim 5.5 \mathrm{~mm}$. The contrast was markedly enhanced in the images, which indicated the presence of multiple neurofibromas (Fig. 1D and E). Electrocardiogram, chest radiograph, liver and kidney function, and the coagulation profile all appeared normal. The patient was revealed to have diabetes during the intial examination following hospitalization. Following preoperative preparation, the patient underwent radical tumor resection of the right kidney under general anesthesia. The sample was dissected following surgery, and the tumor was observed in the middle and upper portions of the right kidney with a size of $\sim 8 \times 7 \times 6 \mathrm{~cm}$. The tumor displayed fish-flesh-like lesions accompanied by necrosis and had metastasized into the surrounding renal pelvis (Fig. 1F).

Postoperative pathological examination confirmed the diagnosis of renal clear cell carcinoma in the right kidney. The tumor did not invade the surrounding adipose tissue or the residual ureter and blood vessels (Fig. 1G).

Hematoxylin and eosin staining was performed. Tissue paraffin sections (slice thickness, $5 \mu \mathrm{m}$ ) were prepared and dewaxed with xylene for $15 \mathrm{~min}$. Sections were then placed in $100 \%$ ethanol for $5 \mathrm{sec}, 95 \%$ ethanol for $5 \mathrm{sec}, 80 \%$ ethanol for $5 \mathrm{sec}$ and $70 \%$ ethanol for $5 \mathrm{sec}$, separately, to hydrate. Subsequently, tissue sections were rinsed with running tap water for $5 \mathrm{sec}$. The tissue sections were then stained with hematoxylin (BA-4097; Baso Biotechnology Co. Ltd., Zhuhai, China) for $8 \mathrm{~min}$, following by rinsing with running tap water for $15 \mathrm{sec}$. The tissue sections were placed in hydrochloric acid alcohol for $5 \mathrm{sec}$, and then rinsed with running tap water for 8 min. Eosin (1\%; BA-4098; Baso Biotechnology Co. Ltd.) was used for staining for $15 \mathrm{sec}$. The tissue sections were placed under running tap water to remove excess dye for $20 \mathrm{sec}$, and then placed in $80 \%$ ethanol for $5 \mathrm{sec}$, $95 \%$ ethanol for $5 \mathrm{sec}$, $100 \%$ ethanol for $10 \mathrm{sec}$ and xylene for $2 \mathrm{~min}$, separately. Finally, the tissue sections were sealed. The aforementioned steps were performed at room temperature. Genetic analysis revealed a mutation in the VHL gene of the patient, c.227_229delTCT (p.Phe76del), which resulted in the deletion of phenylalanine at position 76 of the encoded protein. This genetic analysis result was confirmed by Sanger sequencing (Fig. 1H). Pedigree analysis was performed following surgery (Fig. 1I). Further genetic tests and physical examinations were performed on relatives of the patients with informed consent. The tests included abdominal Doppler ultrasonography and brain MRI. Although all family members had no abnormal findings in genetic tests and physical examinations, follow-up visits were scheduled for all family members. The follow-up for the patient continued until September 2015, at which point the patient was in good condition without recurrence or novel tumor development elsewhere.

Case 2. A 41-year-old male patient was admitted to the Department of Ophthalmology and Endocrinology at the Affiliated Hospital of Zunyi Medical College between 24 September and 5 October 2014 due to blurred vision and 

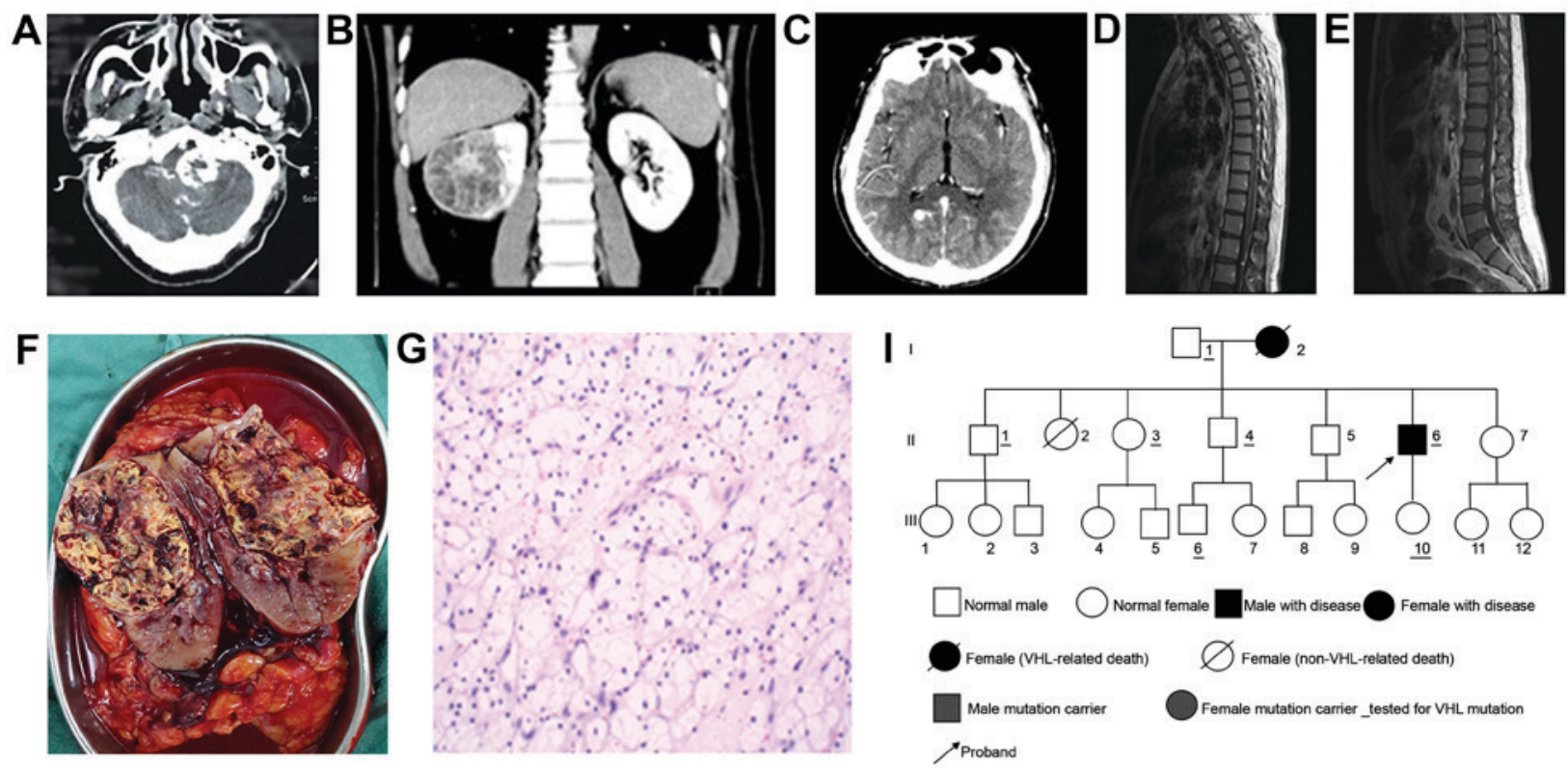

H VHL all.seq (1>12036)

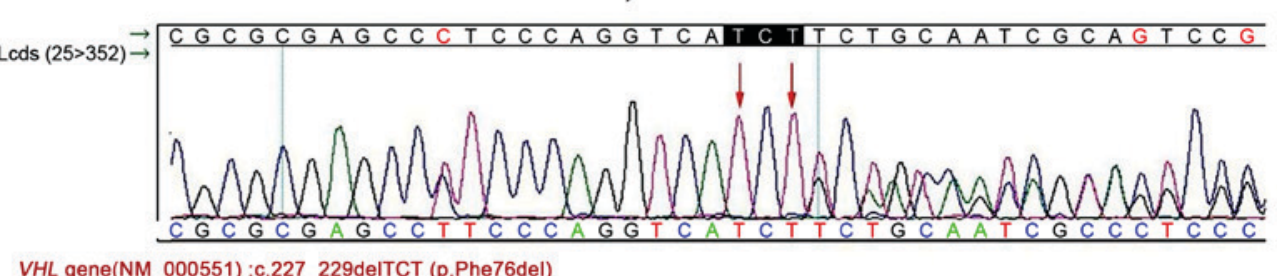

Figure 1. Examination of case 1. (A) Contrast-enhanced CT scan showing lesions in the left side of the brain stem. (B) Contrast-enhanced CT scan indicated a mass in the right kidney $(\sim 64 \times 59 \mathrm{~mm})$ with significant and uneven enhancement. (C) MRI scan indicating malacia in the left cerebellum. Enhanced nodules were observed in the right occipital lobe, cerebellar hemispheres and foramen magnum. The left occipital area was altered following surgery. (D) MRI scan showing multiple enhanced nodules and abnormally enlarged blood vessel clusters in the thoracic spinal canal. (E) MRI scan indicating multiple enhanced nodules in the lumbar spinal canal. (F) Fish-flesh-like alteration, necrosis and metastasis into the renal pelvis and surrounding areas are observed in the tumor. (G) Hematoxylin and eosin staining showing diffusion of a large amount of clear cells in the right kidney, accompanied by cystic formation. The cytoplasm was clear, and the nuclei were of similar size and shape. The nuclei were deep-stained, and nuclear division was rare. Magnification, x200. (H) Genetic test results in case 1 confirmed by Sanger sequencing. (I) Pedigree chart of case 1. CT, computed tomography; MRI, magnetic resonance imaging; VHL, von Hippel-Lindau.

elevated blood glucose level. During hospital admission, adrenal masses were identified on the left and right sides, and the patient was transferred to the Department of Urology on 23 October 2014. Vision was lost in the right eye due to a previous injury. The patient had hypertension and diabetes for $>2$ months. In September 2010, the patient underwent resection of the lesions in the right cerebellar hemisphere. Postoperative pathological analysis confirmed that the lesion was a hemangioblastoma. The mother of the patient was deceased, and the cause was unknown. The mother exhibited a brain tumor and coronary heart disease whilst alive. Multiple nodules were detected in the thyroid gland of the patient's eldest sister (II-1), but were not treated. The rest of the family members were healthy.

Physical examination after admission demonstrated that the body temperature of the patient was $36.8^{\circ} \mathrm{C}$, and the heart rate was 84 beats/min. His respiration rate was 19 times $/ \mathrm{min}$, and blood pressure was $154 / 112 \mathrm{mmHg}$. An old surgical scar was observed in the right occipital area. Opacity of the lens was observed in the right eye, which led to weak vision, while the left eye retained blurry vision. No abnormality was observed in the heart, lung or abdomen. Abdominal CT indicated the presence of irregular bumps in the left (size, $\sim 72 \times 54 \mathrm{~mm}$ ) and right (size, $\sim 92 \times 73 \mathrm{~mm}$ ) adrenal glands. The edge was clear, and the density was uneven. Low-density areas and shadows of calcification were observed. Contrast-enhanced scanning revealed that the solid portion of the mass was markedly enhanced, but the cystic region was not enhanced (Fig. 2A). No swollen lymph nodes were observed in the abdomen, pelvis or retroperitoneal area; therefore, bilateral adrenal pheochromocytoma was considered. Brain CT indicated that the right occipital area was altered following surgery. A cystic low-density area $(34 \times 44 \mathrm{~mm})$ with clear edges was observed in the right cerebellar hemisphere, which was indicative of a cystic lesion or malacia in the right cerebellar hemisphere (Fig. 2B). Cortisol and aldosterone levels were in the normal range. Electrocardiogram, chest radiograph, liver and kidney function, and coagulation profile all appeared normal. Following preoperative preparation, the patient underwent resection of the left adrenal tumor under general anesthesia. The gross sample is presented in Fig. 2C.

Postoperative pathological examination confirmed that the tumor was an adrenal pheochromocytoma (Fig. 2D). Pedigree analysis was performed following surgery (Fig. 2E). Genetic tests and physical examinations were performed for a number of relatives of the patient (who had not succumbed to disease) with informed consent. The same missense mutation, c.499C $>$ T (p.Arg167Trp), in the VHL gene was found in two 

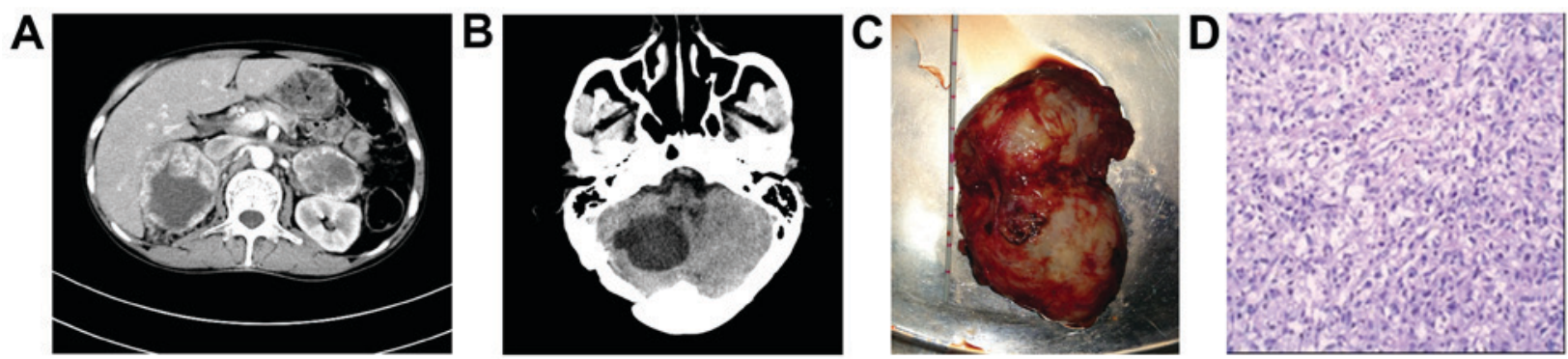

E
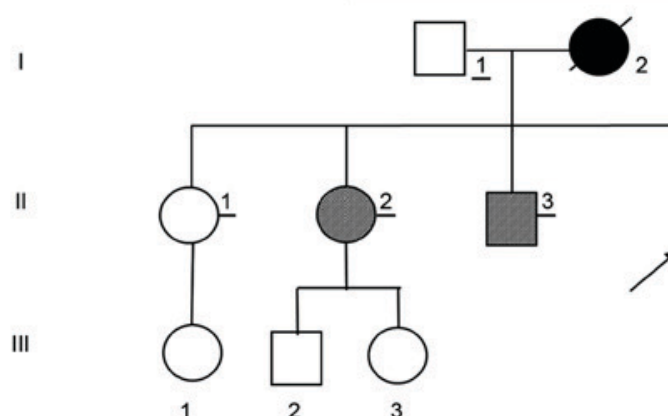

\section{G}

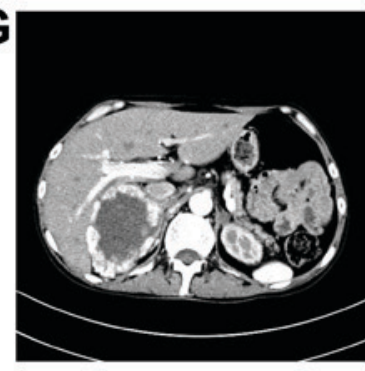

1

2

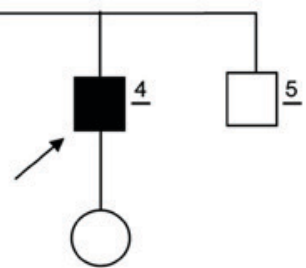

$\underline{4}$

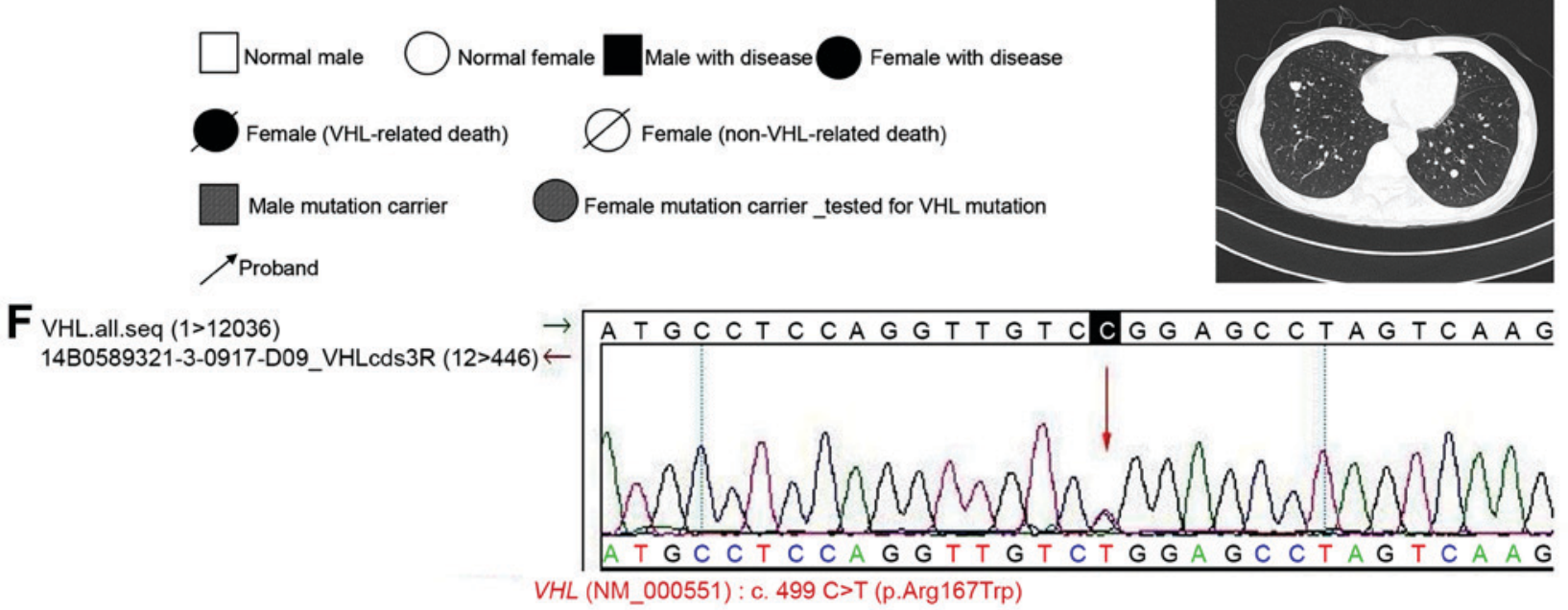

Figure 2. Examination of case 2. (A) CT scan showing nodules in the left and right adrenal glands. Low-density areas and calcification shadows are also observable. A contrast-enhanced scan revealed that the solid portion of the mass was markedly enhanced, but the cystic region was not enhanced. (B) CT scan indicating cystic lesions or malacia in the right cerebellar hemisphere. The right occipital bone was altered following surgery. (C) Gross sample of the tumor obtained following surgery. (D) Hematoxylin and eosin staining indicating proliferation of a large amount of cells with rich cytoplasm in sections of left adrenal gland tissue. The cytoplasm was marginally eosinophilic, weakly stained or transparent and aligned in a nested shape. The nuclei were of varying sizes and were pleomorphic. There were numerous blood vessels with local infiltration of a large amount of eosinophils. Magnification, x200. (E) Pedigree chart of case 2. (F) Confirmation of genetic test results in case 2 by Sanger sequencing. (G) Postoperative abdominal CT indicating an irregular mass $(\sim 92 \times 73 \times 105 \mathrm{~mm})$ in the right adrenal gland, which appeared to be pheochromocytoma and adrenal carcinoma. The left adrenal gland was altered following surgery. $(\mathrm{H}) \mathrm{CT}$ scan revealing multiple metastases in the left and right lungs. The diameter of the largest tumor was $\sim 8.5$ mm. $\mathrm{CT}$, computed tomography; VHL, von Hippel-Lindau.

family members (II-2 and II-3) and the patient, which led to the substitution of arginine with tryptophan at position 167 of the encoded protein. This genetic analysis result was confirmed by Sanger sequencing (Fig. 2F). Physical examinations were also performed, including abdominal Doppler ultrasonography and brain MRI, on several family members. MRI of the brain of the patient's brother (II-3) indicated a nodule in the left cerebellar tonsil with a size of $\sim 9.0 \times 10.5 \mathrm{~mm}$, which indicated the presence of a hemangioblastoma, astrocytoma or ependymoma. However, the diagnosis could not be confirmed. Therefore, patient II-3 was regarded as a carrier of the mutant gene. Doppler ultrasound examination of the patient's eldest sister (II-1) suggested the presence of multiple nodules with calcification and liquefaction in the left and right thyroid glands. The size of the larger nodules was $\sim 34 \times 17 \mathrm{~mm}$ on the left side and $\sim 29 \times 16 \mathrm{~mm}$ on the right side of the thyroid gland. A lymph node (size, $14 \times 4 \mathrm{~mm}$ ) was detected on the right side of the neck, and another lymph node (size, 10x3 mm) was detected on the left side. Spot-like signals corresponding to blood flow were observed inside the node. Another sister of the patient (II-2) carried the mutated VHL gene. However, no VHL-associated lesions were detected, therefore II-2 was considered to be a mutation carrier. Routine follow-ups were suggested.

The daughter and father of the patient declined testing due to personal reasons. The remaining family members had no abnormalities according to the abdominal Doppler ultrasound and brain MRI scan. The family members 
with abnormal results were advised to arrange for routine follow-ups. Due to physical and economic reasons, the patient returned to the Affiliated Hospital of Zunyi Medical College. On 2 June 2015, for resection of the right adrenal gland tumor. Postoperative abdominal CT is presented in Fig. 2G. However, preoperative chest CT indicated that multiple pulmonary metastases were present on the left and right sides (Fig. 2H), which was indicative of malignant pheochromocytoma. Since surgery could not be performed, the patient was advised for a transfer to the Department of Oncology. The patient succumbed following 6 months.

Case 3. A 38-year-old male patient visited the Department of Urology at the Affiliated Hospital of Zunyi Medical College on 27 April 2015, due to the detection of a mass in the right kidney during physical examination. The patient had previously undergone extracorporeal shock wave lithotripsy in February 2010 at Changzheng Hospital (Zunyi, China) for the treatment of kidney stones (details are unknown). There was no history of hypertension, infectious diseases or allergy.

The mother of the patient had pancreatic cancer. She did not undergo surgery and subsequently succumbed to disease. The father of the patient is alive. The eldest sister of the patient (II-1) underwent surgery for a brain tumor in June 2007, and had pancreatic and renal cysts (details are unknown). Another sister (II-2) underwent surgery for breast cancer on the left side in April 2014 and was treated with regular chemotherapy following surgery (details are unknown). The rest of the siblings reported no history of hypertension, diabetes or cancer.

Physical examination after admission demonstrated that the body temperature of the patient was $36.9^{\circ} \mathrm{C}$, and the heart rate was 62 beats/min. The respiration rate was 18 times $/ \mathrm{min}$, and blood pressure was 121/85 $\mathrm{mmHg}$. No abnormalities were observed in the heart, lung or abdomen. Upper abdominal CT revealed the presence of multiple nodules and lumpy low-density areas in the left and right kidneys with uneven density. The diameter of the larger nodule in the right kidney was $\sim 34 \mathrm{~mm}$. Contrast-enhanced imaging indicated that the lesions were enhanced unevenly in a progressive manner with clear edges. Multiple round low-density lesions were observed in the left and right kidneys, and in the pancreas. Contrast-enhanced scanning revealed no significant enhancement. Based on the presence of multiple lesions in the left and right kidneys and the pancreas, as well as the patient's medical history, VHL syndrome was considered as a possible diagnosis (Fig. 3A). Brain CT revealed no abnormalities, but sphenoiditis was observed on the right side. Thoracic MRI revealed a round-shaped mixed signal (diameter, $12.0 \mathrm{~mm}$ ) in the sixth thoracic vertebra. Contrast-enhanced scanning revealed minor enhancement, which appeared to indicate the presence of a hemangioma (Fig. 3B). Lumbar MRI revealed reduced $\mathrm{T} 2$ signals in the $\mathrm{L} 5 / \mathrm{S} 1$ intervertebral disc, which was marginally protruding. Contrast-enhanced scanning did not demonstrate abnormal enhancement. A round long $\mathrm{T} 2$ signal (size, 10x7 mm) was observed in the $\mathrm{S} 2$ horizontal sacral area, which appeared to indicate the presence of a small sacral cyst (Fig. 3C). Electrocardiogram, chest radiograph, liver and kidney function, and coagulation profile all appeared normal. Following preoperative preparation, partial resection of the right kidney was planned, but the patient and his family requested to be discharged.
Follow-up of the patient indicated that the patient underwent partial resection of the right kidney at the West China Hospital Affiliated to Sichuan University (Chengdu, China). Postoperative pathological analysis confirmed that the tumor was a renal carcinoma (clear-cell carcinoma of the right kidney; Fuhrman Grade II) $(6,7)$. The tumor was close to the renal capsule and did not demonstrate significant invasion. The incision area was not invaded by the tumor. Following recovery, the patient returned to the Affiliated Hospital of Zunyi Medical College on 21 September 2015. Bilateral renal CT indicated that the lower part of the right kidney was altered following surgery, and multiple small cystic lesions were detected in the left and right kidneys. The diameter of the largest lesion was $\sim 6 \mathrm{~mm}$. Contrast-enhanced scanning did not reveal marked enhancement. Small cystic lesions were also detected in the pancreas (Fig. 3D). The pedigree of the patient's family was analyzed and established (Fig. 3E). Genetic tests were performed on several relatives with informed consent. The patient and 6 other family members, volunteered to take the VHL genetic test. The tests revealed that the same missense mutation in the VHL gene [c.500G >A (p.Arg167Gln)] was found in the patient and his two sons, which led to a substitution of arginine with glutamine at position 167 of the VHL-encoded protein, and this was confirmed by Sanger sequencing (Fig. 3F). Abdominal ultrasound examination of the patient's two sons revealed no abnormality. MRI of the brain was subsequently planned, but the patient and his two sons declined. Follow-up examinations conducted until October 2015 indicated that the patient was in good condition in general, and no new tumors were identified elsewhere. Follow-up examinations of the other family members remain underway.

\section{Discussion}

VHL syndrome is a rare autosomal dominant genetic disease. The offspring of patients with VHL syndrome have a $50 \%$ chance of inheriting the disease. The gene responsible for this disease is located on chromosome 3p25-26. At the age of 60, the penetrance may exceed $98 \%$ (8). The main clinical manifestations include retinal and CNS hemangioblastoma, which are frequently accompanied by renal clear cell carcinoma, renal cysts, pancreatic tumors, pancreatic cysts and internal lymphoma. In 1859, von Hippel, a German ophthalmologist, for the first time reported two cases of retinal hemangioblastoma with familial inheritance (2,9-11). In 1926, Swiss pathologist Lindau performed a systemic clinical observation of the disease and reported that cerebellar and retinal hemangioblastoma were only parts of the CNS hemangioblastomas, and that the manifestations of these types of hemangioblastoma were associated (2,9-11).

In 1964, Hayden et al (12) first named the disease VHL syndrome, and this nomenclature has been widely recognized. In 1993, Latif et al (3) located the VHL gene on chromosome 3p25-26 and for the first time cloned this gene successfully. A previous study (13) confirmed that the VHL gene is a tumor suppressor that exhibits the classic features of tumor suppressor genes. Mutations and deletions in the VHL gene are the fundamental causes of VHL syndrome (4). Currently, a common diagnostic criterion for VHL syndrome in patients who do 

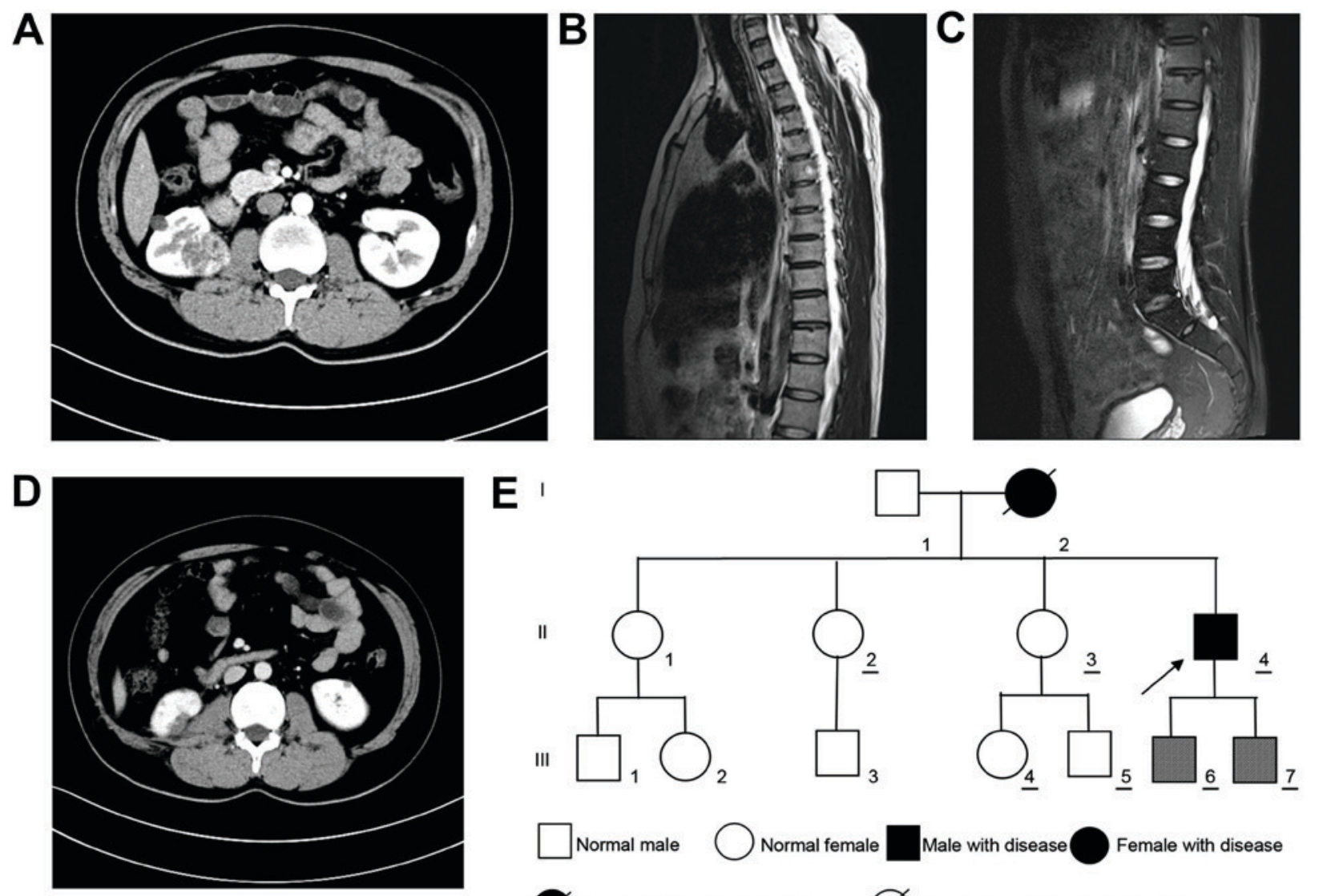

II
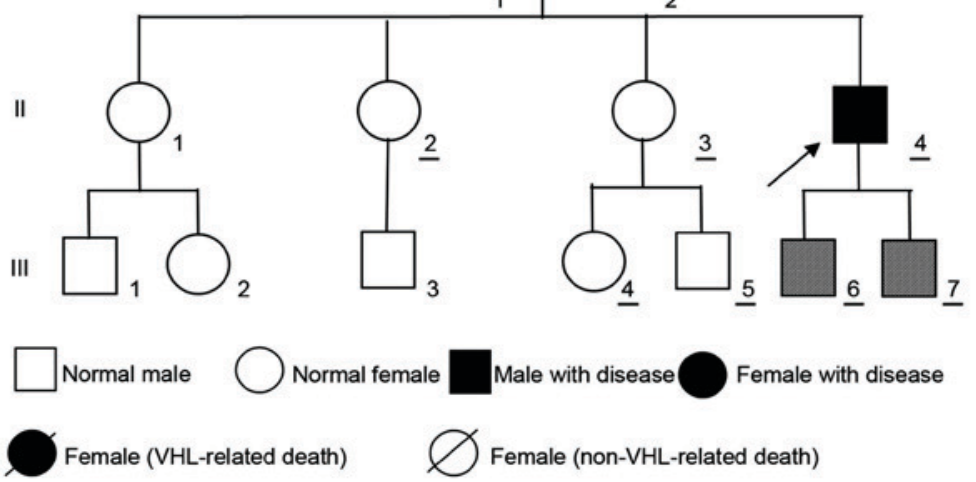

Normal male $\bigcirc$ Normal female $\square$ Male with disease $\bigcirc$ Female with disease

Female (VHL-related death)

Male mutation carrier

Female mutation carrier_tested for VHL mutation

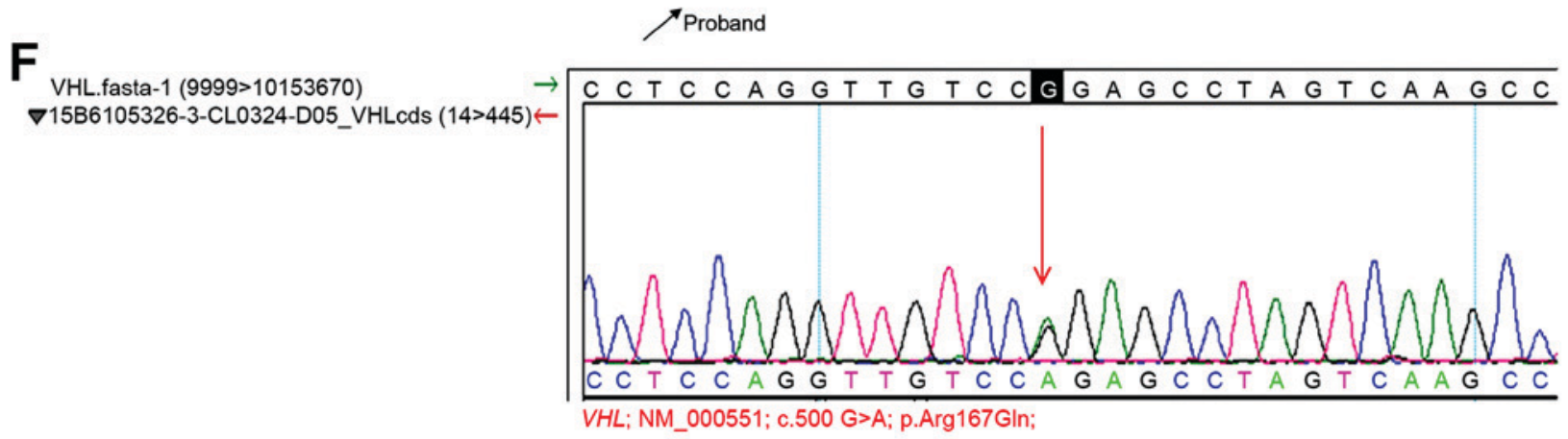

Figure 3. Examination of case 3. (A) CT scan indicating multiple nodules with low-density shadows in left and right kidneys. The larger nodule in the right kidney had a diameter of $\sim 34 \mathrm{~mm}$. Contrast-enhanced scanning showing uneven enhancement in a progressive manner. Bilateral renal and pancreatic multiple cysts were observable. (B) Contrast-enhanced MRI scan showing minor enhancement, which was indicative of a hemangioma. A round mixed signal (diameter, $\sim 12 \mathrm{~mm}$ ) was observable in the sixth thoracic vertebra. (C) MRI scan showing a round long T2 signal in the horizontal sacral area of S2, which indicated the presence of a sacral cyst. (D) Contrast-enhanced CT scanning revealed no marked enhancement. Multiple cystic lesions were observed in the left and right kidneys. The lower part of the right kidney was altered following surgery. (E) Pedigree chart of case 3 . (F) Genetic test results in case 3 confirmed by Sanger sequencing. CT, computed tomography; MRI, magnetic resonance imaging; VHL, von Hippel-Lindau.

not have a family history is that they should exhibit $\geq 2$ of the following characteristic lesions: i) $\geq 2$ retinal, spinal cord or brain hemangioblastomas, or a single hemangioblastoma associated with visceral organ lesions (multiple renal or pancreatic cysts); ii) renal clear cell carcinoma; iii) adrenal or extra-adrenal pheochromocytoma and iv) rare lesions including, internal lymphoma, papillary cystadenoma of the epididymis and broad ligament, and neuroendocrine tumors of the pancreas (14). Patients with a family history of VHL syndrome should have $\geq 1$ of the following lesions: i) Retinal hemangioblastoma, ii) spinal cord or cerebellar hemangioblastoma, iii) adrenal or extra-adrenal pheochromocytoma, iv) renal clear cell carcinoma and v) multiple renal or pancreatic cysts. In patients with atypical clinical manifestations, the VHL genetic test is recommended to exclude the diagnosis of VHL syndrome (14).

From the perspective of clinical practice, diagnosis rates of VHL syndrome in the early stages remain low. This may be due to physicians lacking a thorough understanding of VHL syndrome and therefore overlooking the comprehensive examination of other body systems, family history of the patient and 
genetic tests. Additionally, VHL syndrome is characterized by the presence of multiple tumors in multiple organs; however, the majority of patients with VHL syndrome present with only a single lesion at the time of admission. In the present report, brain hemangioblastoma was detected in cases 1 and 2 when the patients visited the hospital for the first time. According to Glasker et al (15), $19 \%$ of patients with VHL syndrome who are admitted to the hospital for the first time due to neurological symptoms are observed to have multiple hemangioblastomas, and $36 \%$ of patients possess tumor-associated symptoms that are indicative of VHL syndrome. Since the severity of clinical symptoms and the number of tumors affected organs vary, early diagnosis of VHL syndrome is relatively difficult $(1,2)$. In cases 1 and 2 , VHL syndrome was considered when lesions in the visceral organs were detected following resection of the brain hemangioblastomas. The diagnosis could not be confirmed until the results of the genetic tests with the clinical symptoms were combined. According to Conway et al (16), the sensitivity of the VHL genetic test is $86 \%$, and all patients with positive results were confirmed by clinical diagnostic criteria. Therefore, the accuracy of genetic test-based diagnosis is markedly improved compared with clinical manifestation-based diagnosis.

Conway et al (16) found that $67 \%$ of patients with VHL syndrome developed a new CNS tumor within a mean of 2.1 years. In cases 1 and 2 , the mean time for developing a new tumor was 3.5 years. The patient in case 3 remains under close follow-up, and no new tumor has been detected to date. Surgical resection of the tumor remains the main treatment method. In the case of CNS hemangioblastomas, resection is preferred by certain clinicians, while others prefer regular follow-up and decide on surgery when clinical symptoms occur (16). Retinal hemangioblastomas should be removed as soon as possible to avoid loss of vision and other complications. Additionally, VHL syndrome accompanied by pheochromocytoma requires surgery. Partial resection of the adrenal gland may be considered. In patients without hypertension, an $\alpha$-receptor blocker still should be administered (17).

Notably, there are no standard criteria for surgery in cases of VHL syndrome accompanied by renal clear cell carcinoma (17). Duffey et al (18) analyzed 108 VHL syndrome patients with a renal tumor (diameter, $<3 \mathrm{~cm}$ ) and reported that following a mean of 28 months (range, $0-244$ months), no tumor metastasis was observed. Additionally, in VHL syndrome patients $(\mathrm{n}=73)$ with a renal tumor with diameter, $>3 \mathrm{~cm}$, following an average of 72.9 months (range, 0-321 months), metastasis occurred in 20 cases $(27.4 \%)$. Therefore, VHL syndrome patients with a tumor diameter $<3 \mathrm{~cm}$ require close follow-up and monitoring. Abdominal CT and serum creatinine should be examined at least every 6 months. When the diameter of the largest tumor is $>3 \mathrm{~cm}$, surgery should be considered (19-22). Linehan et al (23) suggest that if the size of the tumor is $<3 \mathrm{~cm}$ and it is present on the renal surface, tumor enucleation may be performed. If the tumor size is relatively large $(>4 \mathrm{~cm})$ and is located on one side of the kidney, partial resection should be considered. If the tumor is present in the center of the kidney and is the kidney will be difficult to preserve, radical nephrectomy should be considered (23). The patient in case 1 underwent radical nephrectomy, because the tumor in the right kidney was large and located in the center. Potential invasion into the renal pelvis could not be excluded. In addition, Roupret et al (24) suggested that VHL syndrome-induced renal clear cell carcinoma tends to present as multiple lesions in the left and right kidneys and has a high relapse rate following nephron-sparing surgery. Therefore, thorough elimination of all potential relapses and metastasis may be possible only with bilateral nephrectomy. Following surgery, the patient requires renal replacement therapy, including dialysis and renal transplantation. However, there is evidence that the use of immunosuppressants in patients who require dialysis or renal transplantation following nephrectomy would increase the risk of tumors developing elsewhere (25). Therefore, this treatment plan should only be selected when the left and right kidneys cannot be preserved, and a matched kidney is available for transplantation.

The aim of surgical treatment of VHL syndrome accompanied by renal clear cell carcinoma should be thorough elimination of the tumor and preservation of as much renal tissue as possible. The optimal surgical plan should be selected based on tumor size and site, and the physical conditions of the patient. During postoperative follow-up, relapse in the same kidney or new tumors in the other kidney should be closely monitored (23-25). Based on the diagnosis and treatment in case 2, where VHL syndrome was accompanied by tumors in multiple organs, follow-up surgery for the complete resection of other tumors should be performed as soon as possible following the primary surgery. Even if follow-up surgery is not performed, follow-up should be performed every 3 months. The patient in case 2 was diagnosed with bilateral adrenal pheochromocytoma. Resection of the left adrenal tumor was performed, and postoperative pathological analysis confirmed that it was an adrenal pheochromocytoma. However, the right adrenal tumor was not removed rapidly enough, and the patient did not return for routine follow-up. A total of 8 months later, the patient returned for resection of the right adrenal tumor. However, preoperative chest CT indicated the presence of multiple metastases in the left and right lungs, which was indicative of malignant pheochromocytoma. No surgery could be performed at that time.

Currently, there are certain limitations associated with the surgical treatment of VHL syndrome, as surgical treatment does not prevent tumor relapse and metastasis elsewhere (26). Therefore, postoperative follow-up of the patients and their family members is essential. Emerging investigations of the VHL gene and novel therapeutics, including gene therapy and targeted therapy may be beneficial for patients with VHL syndrome. Early diagnosis, treatment and regular follow-ups are critical for good prognosis of VHL syndrome. Missed diagnosis or misdiagnosis often results from an insufficient understanding of VHL syndrome among physicians (26). Therefore, patients with CNS hemangioblastoma and multiple lesions in visceral organs should be examined carefully, and VHL syndrome should be considered as a potential diagnosis. Genetic tests for VHL should also be conducted to exclude the possibility of VHL syndrome when necessary. Since there is a risk of tumorigenesis with VHL syndrome, lifelong follow-up of the patient is essential. The follow-up should include $(14,26)$ the following measurements: i) urinary catecholamine levels every 6 months; ii) fundus examination annually; iii) CNS MRI every 6 months; and iv) abdominal CT and ultrasound examination annually. 
Since VHL syndrome is an autosomal dominant genetic disorder, pedigree analysis and genetic diagnosis may provide important insights into treatment and prognosis, as well as prenatal and postnatal care. In case 1 , a total of 7 family members volunteered for the genetic test. The proband (II-6) carried a deletion in the VHL gene [c.227_229delTCT (p.Phe76del)] that led to the deletion of phenylalanine at position 76 of the VHL-encoded protein. p.Phe76del blocked the binding of the VHL protein with hypoxia-induced factor- $\alpha$ (HIF- $\alpha$ ), and this affected the stability of HIF- $\alpha$ and induced VHL syndrome (27). The p.Phe76del mutation has been reported to be associated with type I VHL syndrome (27). The remaining family members, including the father of the patient (I-1), did not carry mutations in the VHL gene. However, since the mother of the patient was previously deceased, the authors of the present study hypothesize that this may have been associated with VHL syndrome, and that the patient may have inherited the mutation from his mother.

The patient in case 2 exhibited type II VHL syndrome. Type II VHL syndrome occurs in $\sim 10-20 \%$ of patients with VHL syndrome (1). Sequencing of the VHL gene of the 7 family members revealed the same missense mutation in the VHL gene [c.499C $>\mathrm{T}$ (p.Arg167Trp)] in the proband (II-4), his sister (II-2) and brother (II-3). The mutation resulted in the substitution of arginine at position 167 with tryptophan. Crossey et al (28) and Brauch et al (29) reported that the p.Arg167Trp mutation was closely associated with VHL syndrome. Mutation of the amino acid at position 167 has been reported to increase the risk of pheochromocytoma $(28,29)$. In the present report, the mutation was at position 238. In a study with 469 pedigrees with VHL syndrome across North America, Europe and Japan, Zbar et al (30) observed that p.Arg 167Trp mutation was a hotspot, and the detection rate of this mutation was high in VHL syndrome families with pheochromocytoma. A total of 21 patients with pheochromocytoma in 33 families carrying the p.Arg167Trp mutation were reported. Siu et al (31) performed genetic screening of 9 Chinese pedigrees with VHL syndrome and detected the p.Arg167Trp mutation in a patient with pheochromocytoma. The mother of the patient exhibited a brain tumor and coronary heart disease, and it was likely that she succumbed due to VHL syndrome (31). In the present report, it is possible that the proband (II-4), sister (II-2) and brother (II-3) inherited the mutation from their mother.

In case 3 , a total of 7 members of the family volunteered for the genetic test, and the same missense mutation $[\mathrm{c} .500 \mathrm{G}>\mathrm{A}$ (p.Arg167Gln)] in the VHL gene was detected in the proband (II-4) and his two sons (III-6 and III-7). This mutation led to the substitution of arginine at position 167 with glutamine. Hes et al (32) reported that the p.Arg167Gln mutation was detected in 11 members from 3 typical VHL syndrome pedigrees. Among the 11 members, 4 had renal carcinoma and 3 exhibited renal cysts. Additionally, Ciotti et al (33) found in a controlled study of 43 VHL syndrome patients and 42 healthy controls that the p.Arg167Gln mutation was detected in 2 VHL syndrome patients, where one patient exhibited bilateral renal carcinoma and the other had renal carcinoma, pancreatic cysts and ovarian cysts. The proband of the pedigree also exhibited pancreatic and bilateral renal cysts. Therefore, the authors of the present report hypothesize that the p.Arg167Gln mutation may be associated with cyst formation in visceral organs. However, this hypothesis requires validation in additional cases. In the present report, all members from the three pedigrees who remain alive are under follow-up, particularly those carrying the VHL mutations.

VHL syndrome is a rare autosomal dominant genetic disorder with symptoms that may not be evident, meaning early diagnosis is rare. DNA analysis of VHL genetic mutations is the primary method used to confirm the diagnosis of the disease. The main treatment method of VHL syndrome is surgery. A reasonable surgical strategy should be selected according to the site and number of tumors as well as the physical condition of the patient. Patients with VHL syndrome accompanied by tumors in multiple organs should undergo complete resection of the tumors as soon as possible. Additionally, pedigree analysis and genetic testing are essential. Physicians should pay sufficient attention to VHL syndrome in order to avoid missed diagnosis or misdiagnosis.

\section{Acknowledgements}

The present study was supported by the Science and Technology Fund Project of Guizhou Province [grant no. (2015) 31].

\section{References}

1. Lonser RR, Glenn GM, Walther M, Chew EY, Libutti SK, Linehan WM and Oldfield EH: von Hippel-Lindau disease. Lancet 361: 2059-2067, 2003.

2. Shuin T, Yamazaki I, Tamura K, Kamada M and Ashida S: Recent advances in ideas on the molecular pathology and clinical aspects of Von Hippel-Lindau disease. Int J Clin Oncol 9: 283-287, 2004.

3. Latif F, Tory K, Gnarra J, Yao M, Duh FM, Orcutt ML, Stackhouse T, Kuzmin I, Modi W, Geil L, et al: Identification of the von Hippel-Lindau disease tumor suppressor gene. Science 260: 1317-1320, 1993.

4. Kim W and Kaelin WG Jr: The von Hippel-Lindau tumor suppressor protein: New insights into oxygen sensing and cancer. Curr Opin Genet Dev 13: 55-60, 2003.

5. McNeill A, Rattenberry E, Barber R, Killick P, MacDonald F and Maher ER: Genotype-phenotype correlations in VHL exon deletions. Am J Med Genet A 149A: 2147-2151, 2009.

6. Patard JJ, Leray E, Rioux-Leclercq N, Cindolo L, Ficarra V, Zisman A, De La Taille A, Tostain J, Artibani W, Abbou CC, et al: Prognostic value of histologic subtypes in renal cell carcinoma: A multicenter experience. J Clin Oncol 23: 2763-2771, 2005.

7. Leibovich BC,Cheville JC, Lohse CM,Zincke H, Frank I, Kwon ED, Merchan JR and Blute ML: A scoring algorithm to predict survival for patients with metastatic clear cell renal cell carcinoma: A stratification tool for prospective clinical trials. J Urol 174: 1759-1763, 2005.

8. Vortmeyer AO, Choo D, Pack S, Oldfield E and Zhuang Z: VHL gene inactivation in an endolymphatic sac tumor associated with von Hippel-Lindau disease. Neurology 55: 460, 2000.

9. Neumann HP and Wiestler OD: Clustering of features of von Hippel-Lindau syndrome: Evidence for a complex genetic locus. Lancet 337: 1052-1054, 1991.

10. Weil RJ, Vortmeyer AO, Zhuang Z, Pack SD, Theodore N, Erickson RK and Oldfield EH: Clinical and molecular analysis of disseminated hemangioblastomatosis of the central nervous system in patients without von Hippel-Lindau disease. Report of four cases. J Neurosurg 96: 775-787, 2002.

11. Kanno H, Yamamoto I, Yoshida M and Kitamura H: Meningioma showing VHL gene inactivation in a patient with von Hippel-Lindau disease. Neurology 60: 1197-1199, 2003.

12. Hayden MG, Gephart R, Kalanithi P and Chou D: Von Hippel-Lindau disease in pregnancy: A brief review. J Clin Neurosci 16: 611-613, 2009.

13. Bader HL and Hsu T: Systemic VHL gene functions and the VHL disease. FEBS Lett 586: 1562-1569, 2012. 
14. Maher ER, Neumann HP and Richard S: von Hippel-Lindau disease: A clinical and scientific review. Eur J Hum Genet 19: 617-623, 2011

15. Glasker S, Bender BU, Apel TW, Natt E, van Velthoven V, Scheremet R, Zentner J and Neumann HP: The impact of molecular genetic analysis of the VHL gene in patients with haemangioblastomas of the central nervous system. J Neurol Neurosurg Psychiatry 67: 758-762, 1999.

16. Conway JE, Chou D, Clatterbuck RE, Brem H, Long DM and Rigamonti D: Hemangioblastomas of the central nervous system in von Hippel-Lindau syndrome and sporadic disease. Neurosurgery 48: 55-63, 2001.

17. Benhammou JN, Boris RS, Pacak K, Pinto PA, Linehan WM and Bratslavsky G: Functional and oncologic outcomes of partial adrenalectomy for pheochromocytoma in patients with von Hippel-Lindau syndrome after at least 5 years of followup. J Urol 184: 1855-1859, 2010.

18. Duffey BG, Choyke PL, Glenn G, Grubb RL, Venzon D, Linehan WM and Walther MM: The relationship between renal tumor size and metastases in patients with von Hippel-Lindau disease. J Urol 172: 63-65, 2004.

19. Steinbach F, Novick AC, Zincke H, Miller DP, Williams RD, Lund G, Skinner DG, Esrig D, Richie JP, deKernion JB, et al: Treatment of renal cell carcinoma in von Hippel-Lindau disease: A multicenter study. J Urol 153: 1812-1816, 1995.

20. Ghavamian R, Cheville JC, Lohse CM, Weaver AL, Zincke H and Blute ML: Renal cell carcinoma in the solitary kidney: An analysis of complications and outcome after nephron sparing surgery. J Urol 168: 454-459, 2002.

21. Albers P: Treatment approaches in renal cell carcinoma: past, present and future perspectives. Eur Urol Suppl 7: 36-45, 2008 .

22. Ploussard G, Droupy S, Ferlicot S, Ples R, Rocher L, Richard S and Benoit G: Local recurrence after nephron-sparing surgery in von Hippel-Lindau disease. Urology 70: 435-439, 2007.

23. Linehan WM, Rubin JS and Bottaro DP: VHL loss of function and its impact on oncogenic signaling networks in clear cell rena cell carcinoma. Int J Biochem Cell Biol 41: 753-756, 2009.

24. Roupret M, Hopirtean V, Mejean A, Thiounn N, Dufour B, Chretien Y, Chauveau D and Richard S: Nephron sparing surgery for renal cell carcinoma and von Hippel-Lindau's disease: A single center experience. J Urol 170: 1752-1755, 2003.
25. Marcos HB, Libutti SK, Alexander HR, Lubensky IA, Bartlett DL, Walther MM, Linehan WM, Glenn GM and Choyke PL: Neuroendocrine tumors of the pancreas in von Hippel-Lindau disease: Spectrum of appearances at CT and MR imaging with histopathologic comparison. Radiology 225: 751-758, 2002.

26. Frantzen C, Links T and Giles R: Von Hippel-Lindau Disease. Seattle (WA): University of Washington, Seattle, 2000.

27. Jia D, Tang B, Shi Y, Wang J, Sun Z, Chen Z, Zhang L, Xia K and Jiang H: A deletion mutation of the VHL gene associated with a patient with sporadic von Hippel-Lindau disease. J Clin Neurosci 20: 842-847, 2013.

28. Crossey PA, Richards FM, Foster K, Green JS, Prowse A, Latif F, Lerman MI, Zbar B, Affara NA, Ferguson-Smith MA, et al: Identification of intragenic mutations in the von Hippel-Lindau disease tumour suppressor gene and correlation with disease phenotype. Hum Mol Genet 3: 1303-1308, 1994.

29. Brauch H, Kishida T, Glavac D, Chen F, Pausch F, Höfler H, Latif F, Lerman MI, Zbar B and Neumann HP: Von Hippel-Lindau (VHL) disease with pheochromocytoma in the Black Forest region of Germany: Evidence for a founder effect. Hum Genet 95: 551-556, 1995.

30. Zbar B, Kishida T, Chen F, Schmidt L, Maher ER, Richards FM, Crossey PA, Webster AR, Affara NA, Ferguson-Smith MA, et al: Germline mutations in the Von Hippel-Lindau disease (VHL) gene in families from North America, Europe, and Japan. Hum Mutat 8: 348-357, 1996.

31. Siu WK, Ma RC, Lam CW, Mak CM, Yuen YP, Lo FM, Chan KW, Lam SF, Ling SC, Tong SF, et al: Molecular basis of von Hippel-Lindau syndrome in Chinese patients. Chin Med J (Engl) 124: 237-241, 2011.

32. Hes FJ, van der Luijt RB, Janssen AL, Zewald RA, de Jong GJ, Lenders JW, Links TP, Luyten GP, Sijmons RH, Eussen HJ, et al: Frequency of Von Hippel-Lindau germline mutations in classic and non-classic Von Hippel-Lindau disease identified by DNA sequencing, Southern blot analysis and multiplex ligation-dependent probe amplification. Clin Genet 72: 122-129, 2007.

33. Ciotti P, Garuti A, Gulli R, Ballestrero A, Bellone E and Mandich P: Germline mutations in the von Hippel-Lindau gene in Italian patients. Eur J Med Genet 52: 311-314, 2009.

This work is licensed under a Creative Commons Attribution-NonCommercial-NoDerivatives 4.0 International (CC BY-NC-ND 4.0) License. 